\title{
Sparse channel recovery with inter-carrier interference self-cancellation in OFDM
}

\author{
HU Jiansheng ${ }^{1,2, *}$, SONG Zuxun ${ }^{1}$, GUO Shuxia ${ }^{1}$, ZHANG Qian², and SHUI Dongdong ${ }^{2}$ \\ 1. School of Electronics and Information, Northwestern Polytechnical University, Xi' an 710072, China; \\ 2. School of Information Engineering, Engineering Universtiy of Chinese People's Armed Police Force, Xi' an 710086, China
}

\begin{abstract}
A new sparse channel estimation method of orthogonal frequency division multiplexing (OFDM) system based on intercarrier interference (ICI) self-cancellation is investigated. Firstly, based on the characteristic that the $\mathrm{ICl}$ generated by a subcarrier to the two adjacent subcarriers is approximately equal, a data pair with opposite sign and equal magnitude is modulated onto two adjacent subcarriers as pilot pair to eliminate the effect of $\mathrm{ICI}$ on pilots. Secondly, a new OFDM channel estimation model based on linear time-varying (LTV) model and compressed sensing (CS) is constructed, which obtains the mean of the gains of the multipath. Finally, a pilot pair optimization algorithm based on two layers loop is used to realize the minimization of the mutual coherence of the measurement matrix. For time-varying channel scenes with different numbers or delay of multipath and maximum Doppler frequency shift, the performances of several channel estimation methods are verified by simulation. The result shows that the new method has obvious advantage in both the performance of the channel estimation and the spectral efficiency.
\end{abstract}

Keywords: orthogonal frequency division multiplexing (OFDM), channel estimation, compressed sensing (CS), inter-carrier interference (ICl), pilot design.

DOI: $10.21629 / J S E E .2018 .04 .02$

\section{Introduction}

There are two main problems for orthogonal frequency division multiplexing (OFDM) system channel estimation in high speed environment [1,2]. Firstly, the value of channel impulse response (CIR) is not constant during one OFDM symbol period, and the number of parameters to be estimated is very huge. Secondly, the orthogonality between the subcarriers is destroyed due to the Doppler frequency shift, which causes inter-carrier interference (ICI).

For the first issue, the channel matching model is usually used to reduce the number of parameters to be estimated in time-varying channels, such as linear time-varying (LTV)

\footnotetext{
Manuscript received June 07, 2017.

*Corresponding author.

This work was supported by the National Natural Science Foundation of China (61571368).
}

channel model and basis expansion model (BEM). The LTV model is relatively simple, and when the normalized maximum Doppler frequency shift is less than 0.2, the performance of LTV is perfect and suitable for most mobile communication occasions [3-6]. The LTV model was first proposed by Mostofi in [3]. The main idea of the method is that the variation of each CIR is assumed to be a linear approximation with a constant slope in one OFDM symbol, then the averages and slopes of each multi-path is estimated by equal space pilots during three successive OFDM symbols. Because the pilots are affected by the ICI from the unknown data, the performance of channel estimation is not ideal. As for the second issue, there are several main ICI cancellation methods based on the LTV model, including iterative method [4], window-LTV [5] and ICI self-cancellation [6,7]. However, the iteration increases the amount of computation and reduces the timeliness. The window function is added into the OFDM system, which reduces the ICI of out band, but needs to change the structure of OFDM system. The ICI self-cancellation method in [6] and [7], uses ' 1 ' and ' -1 ' as two adjacent pilots to realize the ICI self-cancellation, but introduces notable performance degrade in long-delay channel, as shown in Section 5. In addition, the common drawbacks of these methods include: the pilot number cannot be smaller than the channel length, and the system frequency utilization is lower. And the threshold is used to determine the effective taps, and the error is large under low signal to noise ratio (SNR).

In recent years, a large number of experimental results show that the wireless multipath channel with scattering environment distribution has obvious sparse characteristics and strong temporal correlation $[8,9]$. The number of multipath is far smaller than the length of the channel. The change of multipath delay can be considered as constant during one or even a few OFDM symbols $[10,11]$. The compressed sensing (CS) theory can be applied in the channel estimation to gain better performance, and the ICI 
can also be reduced through reasonable pilot design, which is called sparse recovery of time-varying channel [12-14]. However, the computation of sparse recovery based on the BEM is still very large. And the research into the sparse channel estimation based on the LTV model is very little. Finally, the pilot pattern design is important for the reconstruction of sparse channel, however, at present, the researches on this problem are all aimed at the untimevarying channel, and only consider the mutual coherence of the measurement matrix, ignoring the influence of ICI, which is not suitable for sparse reconstruction of timevarying channels [14-16].

In view of the above problems, this paper analyzes the characteristics of ICI, uses the special pilot pattern to eliminate the ICI from unknown data. And the estimation of the mean values of multi-path gains is converted to a sparse reconstruction problem.

The rest of this paper is organized as follows: The signal model is described in Section 2. In Section 3, the ICI characteristic of OFDM system is analyzed and an ICI mitigation based on pilot pair is discussed. Section 4 presents the new sparse recovery model and the pilot optimization algorithm. In Section 5, the simulation results are given. Finally, Section 6 concludes this paper.

\section{System model}

\subsection{OFDM model}

We assume an OFDM system with $N$ subcarriers. $x[n]$ and $y[n]$ denote the transmitted symbol and the received symbol in time domain respectively, and $n \in[0, N-1]$. Then the received OFDM symbol $y[n]$ after cyclic prefix (CP) removal is given by

$$
y[n]=\sum_{l=0}^{L-1} h[n, l] x[n-l]+w[n]
$$

where $h[n, l]$ represents the complex zero-mean unitvariance CIR tap at lag $l(l=0,1, \ldots, L-1)$ and time instant $n$, and $L$ is the maximum multipath delay. $w[n]$ is the additive noise. Taking the discrete Fourier transform (DFT) of (1), we can get

$$
Y_{k}=G_{k, k} X_{k}+\sum_{m=0, m \neq k}^{N-1} G_{k, m} X_{m}+W_{k}
$$

where $X_{k}$ and $Y_{k}$ denote the modulated signal and demodulated signal of the $k$ th carrier respectively, $G_{k, k} X_{k}$ is the ideal received signal in the case of ICI-free channel, $\sum_{m=0, m \neq k}^{N-1} G_{k, m} X_{m}$ is the one in the case of ICI, and $W_{k}$ is addictive noise. $G_{k, m}(m \neq k)$ and $G_{k, k}$ are represented respectively as

$$
\begin{gathered}
G_{k, m}=\frac{1}{N} \sum_{n=0}^{N-1} \sum_{l=0}^{L-1} h[n, l] \mathrm{e}^{-\mathrm{j} 2 \pi n(k-m) / N} \mathrm{e}^{-\mathrm{j} 2 \pi m l / N} \\
G_{k, k}=\sum_{l=0}^{L-1} h^{\mathrm{ave}}(l) \mathrm{e}^{-\mathrm{j} 2 \pi k l / N}
\end{gathered}
$$

where $h^{\text {ave }}(l)=\frac{1}{N} \sum_{n=0}^{N-1} h[n, l]$ denotes the time average of $h[n, l]$ over one OFDM symbol. The $h^{\text {ave }}(l)$ for all paths can be denoted as $\boldsymbol{h}^{\text {ave }}=\left[h^{\text {ave }}(0), h^{\text {ave }}(1), \ldots\right.$, $\left.h^{\text {ave }}(L-1)\right]$. Because the channel is sparse, most of components of $\boldsymbol{h}^{\text {ave }}$ are zero, or approximately zero, which means $\boldsymbol{h}^{\text {ave }}$ is a sparse vector.

\subsection{LTV channel model}

For the LTV channel model, CIR can be equivalent to a linear change during one OFDM symbol period. The LTV model can be divided into two kinds: single line and double line, also called piece-wise. The former is to estimate the slope by using the CP of the current OFDM symbol, and the latter is using three consecutive symbols. In contrast, the model error of the latter is much smaller, and the performance is much higher, which is more widely used, shown as Fig. 1.

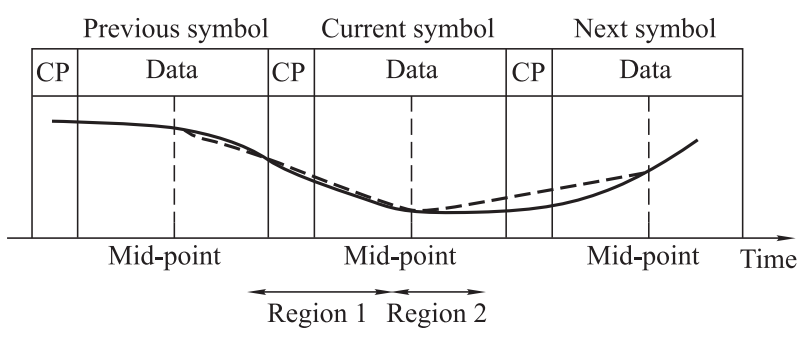

Fig. 1 Piece-wise LTV model

In the piece-wise LTV model, $h[n, l]$ of current symbol can be denoted as

$$
\left\{\begin{aligned}
h[n, l] & =h\left[\frac{N}{2}-1, l\right]+s_{l}^{1} \times\left(n-\frac{N}{2}+1\right), \\
n & \in\left[0, \frac{N}{2}-1\right] \\
h[n, l] & =h\left[\frac{N}{2}-1, l\right]+s_{l}^{2} \times\left(n-\frac{N}{2}+1\right), \\
n & \in\left[\frac{N}{2}, N-1\right]
\end{aligned}\right.
$$

where $s_{l}^{1}$ and $s_{l}^{2}$ are the slopes of region 1 and region 2 . 
They can be obtained by using adjacent symbols.

$$
\left\{\begin{array}{c}
s_{l}^{1}=\frac{h\left[\frac{N}{2}-1, l\right]-h^{\text {previous }\left[\frac{N}{2}-1, l\right]}}{N} \\
s_{l}^{2}=\frac{h^{\text {next }\left[\frac{N}{2}-1, l\right]-h\left[\frac{N}{2}-1, l\right]}}{N}
\end{array}\right.
$$

Approximation error would be minimized, if we approximate $h\left[\frac{N}{2}-1, l\right]$ with the estimation of $h^{\text {ave }}(l)$ [3]. That is $h\left[\frac{N}{2}-1, l\right] \approx h^{\text {ave }}(l)$, so the relationship between the received signal and the sent signal in frequency domain can also be expressed as

$$
\boldsymbol{Y}=\boldsymbol{H}^{\text {ave }} \boldsymbol{X}+\left(\boldsymbol{C}^{s 1} \boldsymbol{H}^{s 1}+\boldsymbol{C}^{s 2} \boldsymbol{H}^{s 2}\right) \boldsymbol{X}+\boldsymbol{W}
$$

where $\boldsymbol{Y}=\left[Y_{0}, Y_{1}, \ldots, Y_{N-1}\right]^{\mathrm{T}}, \boldsymbol{X}=\left[X_{0}, X_{1}, \ldots\right.$, $\left.X_{N-1}\right]^{\mathrm{T}}, \quad \boldsymbol{W}=\left[W_{0}, W_{1}, \ldots, W_{N-1}\right]^{\mathrm{T}}, \quad \boldsymbol{H}^{\text {ave }}=$ $\operatorname{diag}\left\{\operatorname{FFT}\left(\left[h^{\text {ave }}(0), \ldots, h^{\text {ave }}(L-1), 0, \ldots, 0\right]\right)\right\}, \boldsymbol{H}^{s 1}=$ $\operatorname{diag}\left\{\operatorname{FFT}\left(\left[s_{0}^{1}, s_{1}^{1}, \ldots, s_{L-1}^{1}, 0, \ldots, 0\right]\right)\right\} . \quad \boldsymbol{H}^{s 2}=$ $\operatorname{diag}\left\{\operatorname{FFT}\left(\left[s_{0}^{2}, s_{1}^{2}, \ldots, s_{L-1}^{2}, 0, \ldots, 0\right]\right)\right\} . \boldsymbol{C}^{s 1}$ and $\boldsymbol{C}^{s 2}$ are fixed matrixes, and the special values are shown in [3].

Obviously, it is a key issue to estimate $h^{\text {ave }}(l)$ of one OFDM symbol for the channel estimation based on the LTV model.

\section{ICI characteristic and self-cancellation method}

\subsection{ICI characteristic of OFDM system}

When the angular spread is small for non-resolvable components of a channel, the discrete-time CIR of a timevarying multipath channel $[6,18]$ can be also expressed as

$$
h[n, l]=\sum_{u=0}^{L-1} a_{u} \mathrm{e}^{\frac{\mathrm{j} 2 \pi \Delta f_{u} n}{N}} \delta(l-u)
$$

where $a_{u}$ is the complex number for the $u$ th resolvable multipath (tap), $\Delta f_{u}$ is the corresponding Doppler frequency normalized by one subcarrier spacing. By substituting (8) into (3), we can get

$$
\begin{gathered}
G_{k, m}=\frac{1}{N} \sum_{n=0}^{N-1} \sum_{l=0}^{L-1} h[n, l] \mathrm{e}^{-\mathrm{j} 2 \pi n(k-m) / N} \mathrm{e}^{-\mathrm{j} 2 \pi m l / N}= \\
\frac{1}{N} \sum_{l=0}^{L-1}\left\{a_{l} \frac{\sin \left[\pi\left(m+\Delta f_{l}-k\right)\right]}{N \sin \left[\frac{\pi}{N}\left(m+\Delta f_{l}-k\right)\right]} .\right. \\
\left.\mathrm{e}^{-\mathrm{j} 2 \pi m u / N} \mathrm{e}^{\mathrm{j} \frac{\pi(N-1)}{N}\left(m+\Delta f_{l}-k\right)}\right\} .
\end{gathered}
$$

If we only take the $l$ th tap into consideration, and use $S_{l}(k, m)$ to denote the ICI coefficient between the $k$ th subcarrier and the $m$ th subcarrier, then the $G_{k, m}$ can be taken as a linear combination of $S_{l}(k, m)(l=0,1, \ldots, L-1)$.

$$
\begin{gathered}
S_{l}(k, m)=\frac{\sin \left[\pi\left(m+\Delta f_{l}-k\right)\right]}{N \sin \left[\frac{\pi}{N}\left(m+\Delta f_{l}-k\right)\right]} . \\
\mathrm{e}^{-\mathrm{j} 2 \pi m l / N} \mathrm{e}^{\mathrm{j} \frac{\pi(N-1)}{N}\left(m+\Delta f_{l}-k\right)}
\end{gathered}
$$

Obviously, the amplitude of $S_{l}(k, m)$ only depends on $|m-k|$. Fig. 2 shows $\left|S_{l}(k, m)\right|$ for different $m$ and $k$ in the case of $\Delta f_{l}=0.1$.

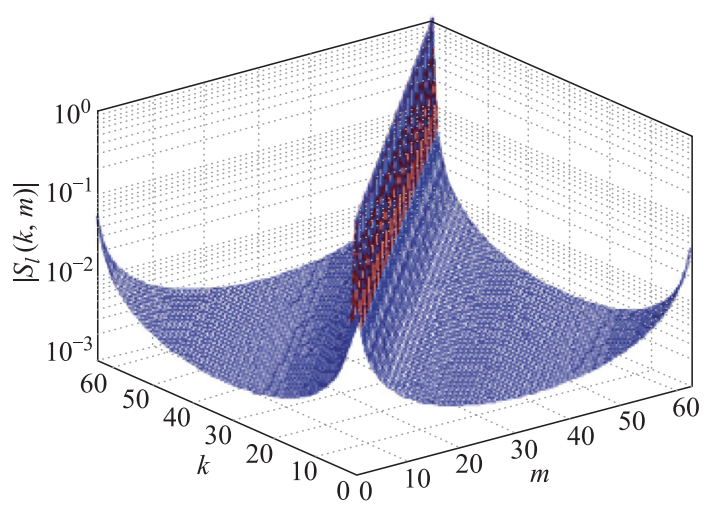

(a) All values of $k$ and $m$, for $N=64$ and $\Delta f_{l}=0.1$

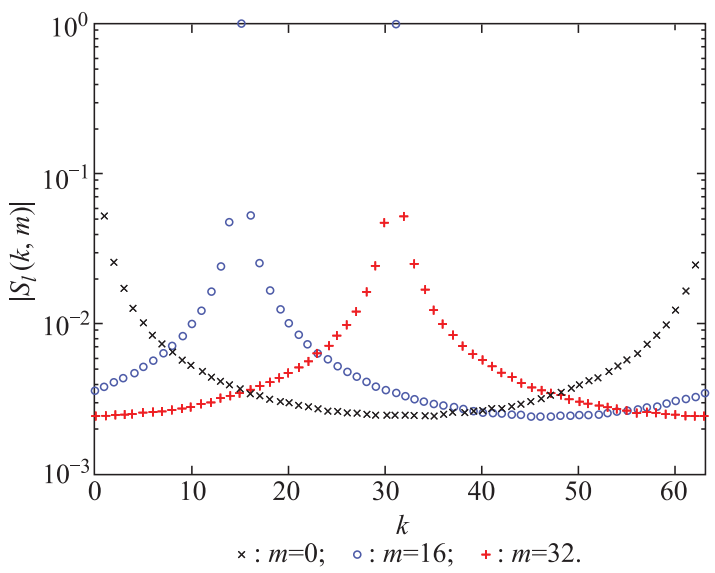

(b) An example for a given $m=0,16$ and 32

Fig. 2 Value of $\left|S_{l}(k, m)\right|$ for different $m$ and $k$

According to Fig. 2, we can draw two conclusions: First, the ICI from a certain subcarrier mainly affects its adjacent tones. Second, for the majority of $m-k$, the value of $\left|S_{l}(k, m)\right|$ changes little. For a given $m$ and most $k,\left|S_{l}(k, m)\right| \approx\left|S_{l}(k+1, m)\right|$. Because the $G_{k, m}$ can be seen as a linear combination of $\left|S_{l}(k, m)\right|$, and due to the sparsity of wireless channel, for $l=0,1, \ldots, L-1$, most of $a_{l}$ is zero, then the ICI generated by a subcarrier to the two adjacent subcarriers is approximately equal. That is, for a given $m$ and most of $k, G_{k, m} \approx G_{k+1, m}$. 


\subsection{ICI mitigation based on pilots pair}

Based on the analysis of Section 3.1, when we design the pilot pattern, a data pair $(-1,1)$ is modulated onto two adjacent subcarriers $\left(k_{P}, k_{P}+1\right)$ as a pilot pair, that is to say, $X\left(k_{P}\right)=-X\left(k_{P}+1\right), k_{P} \in \boldsymbol{P}$, where $\boldsymbol{P}=\left\{P_{1}, P_{2}, \ldots, P_{N P}\right\}$ is the pilots location set, and $N_{P}$ is the toll number of pilots, $0 \leqslant P_{1}, P_{1}+1=P_{2}$, $P_{3}+1=P_{4}, \ldots, P_{N_{P-1}}+1=P_{N_{P}}, P_{N_{P}} \leqslant N-1$. Then according to (2), the pilots received on the $k_{P}$ th and $\left(k_{P}+1\right)$ th sub-carrier can be expressed as follows:

$$
\begin{gathered}
Y_{P}\left(k_{P}\right)=X\left(k_{P}\right) G_{k_{P}, k_{P}}+ \\
\sum_{m=0, m \neq k_{P}}^{N-1} X(m) G_{k_{P}, m}+W\left(k_{P}\right) \\
Y_{P}\left(k_{P}+1\right)=X\left(k_{P}+1\right) G_{k_{P}+1, k_{P}+1}+ \\
\sum_{m=0, m \neq k_{P}+1}^{N-1} X(m) G_{k_{P}+1, k_{P}+1}+W\left(k_{P}+1\right) .
\end{gathered}
$$

Through (11) and (12), we can get

$$
\begin{gathered}
Y_{P}\left(k_{P}\right)-Y_{P}\left(k_{P}+1\right)=X\left(k_{P}\right)\left[G_{k_{P}, k_{P}}+G_{k_{P}+1, k_{P}+1}\right]+ \\
I C I_{k_{P}}-I C I_{k_{P}+1}+W\left(k_{P}\right)-W\left(k_{P}+1\right)
\end{gathered}
$$

where $I C I_{k_{P}}=\sum_{m=0, l \neq k_{P}}^{N-1} X(m) G_{k_{P}, m}, I C I_{k_{P}+1}=$ $\sum_{m=0, m \neq k_{P}+1}^{N-1} X(m) G_{k_{P}+1, m}$. Based on the conclusion of Section 3.1, for a given $m$ and most of $k, G_{k, m} \approx G_{k+1, m}$, $I C I_{k p}-I C I_{k p+1} \approx 0$. Obviously, compared with (11) or (12), the effect of ICI on pilots in (13) is much less, and the performance of channel estimation will be improved.

\section{A new channel sparse recovery method}

\subsection{New sparse recovery model based on pilot pair}

For (13), the classical channel estimation model based on CS theory, such as in [12-14], is not suitable. Therefore, we must build a new channel sparse recovery model.

Because the ICI is very little in (13), it can be treated as noise. Assuming $W^{\prime}\left(k_{P}, k_{P}+1\right)=I C I_{k_{P}}-I C I_{k_{P}+1}+$ $W\left(k_{P}\right)-W\left(k_{P}+1\right)$, substituting (3) and (4) into (13), we can get

$$
\begin{gathered}
Y_{P}\left(k_{P}\right)-Y_{P}\left(k_{P}+1\right)=X\left(k_{P}\right)\left[\sum_{l=0}^{L-1} h^{\mathrm{ave}}(l) \mathrm{e}^{-\mathrm{j} 2 \pi k_{P} l / N}+\right. \\
\left.\sum_{l=0}^{L-1} h^{\mathrm{ave}}(l) \mathrm{e}^{-\mathrm{j} 2 \pi\left(k_{P}+1\right) l / N}\right]+W^{\prime}\left(k_{P}, k_{P}+1\right) .
\end{gathered}
$$

Based on the pilot pair design principle of Section 3.2, we divide the pilot location set $\boldsymbol{P}$ into two subsets, $\boldsymbol{P}_{\text {odd }}=$ $\left\{P_{1}, P_{3}, \ldots, P_{N_{P-1}}\right\}$ and $\boldsymbol{P}_{\text {even }}=\left\{P_{2}, P_{4}, \ldots, P_{N_{P}}\right\}$. Then (14) can be written in matrix notation as

$$
\begin{gathered}
\boldsymbol{Y}_{\text {odd }}-\boldsymbol{Y}_{\text {even }}=\boldsymbol{X}_{\text {odd }} \boldsymbol{F}_{\text {odd }} \boldsymbol{h}^{\text {ave }}-\boldsymbol{X}_{\text {even }} \boldsymbol{F}_{\text {even }} \boldsymbol{h}^{\text {ave }}+ \\
\boldsymbol{W}_{\text {odd }}-\boldsymbol{W}_{\text {even }}
\end{gathered}
$$

where for $i \in \boldsymbol{P}_{\text {odd }}$ and $j \in \boldsymbol{P}_{\text {even }}, \boldsymbol{X}_{\text {odd }}$ and $\boldsymbol{X}_{\text {even }}$ are both the subset of $\boldsymbol{X}$, and written as diagonal matrix, whose diagonal elements are all $X_{i}$ and $X_{j}$, respectively. $\boldsymbol{Y}_{\text {odd }}$ and $\boldsymbol{Y}_{\text {even }}$ are column vectors, which are composed of all $Y_{i}$ and $Y_{j}$, respectively. $\boldsymbol{W}_{\text {odd }}$ and $\boldsymbol{W}_{\text {even }}$ are column vectors, which are composed of all $W_{i}$ and $W_{j}$, respectively. $\boldsymbol{F}_{\text {odd }}$ is a sub-matrix selected by the row indices $\boldsymbol{P}_{\text {odd }}$ and column indices $[0,1, \ldots, L-1]$ from $\boldsymbol{F}_{\mathrm{N} \times \mathrm{N}}, \boldsymbol{F}_{\text {odd }}$ is a submatrix selected by the row indices $\boldsymbol{P}_{\text {even }}$ and column indices $[0,1, \ldots, L-1]$ from $\boldsymbol{F}_{N \times N}$, where $\boldsymbol{F}_{N \times N}$ is the standard DFT matrix.

Assume $X_{i}=1, i \in \boldsymbol{P}_{\mathrm{odd}}$, and $X_{j}=-1, j \in \boldsymbol{P}_{\text {even }}$, then (15) can be simplified as

$$
\boldsymbol{Y}_{\text {odd }}-\boldsymbol{Y}_{\text {even }}=\boldsymbol{A} \boldsymbol{h}^{\text {ave }}+\boldsymbol{W}^{\prime}
$$

where $\boldsymbol{Y}^{\prime}=\boldsymbol{Y}_{\text {odd }}-\boldsymbol{Y}_{\text {even }}, \boldsymbol{A}=\boldsymbol{F}_{\text {odd }}-\boldsymbol{F}_{\text {even }}$ and $\boldsymbol{W}^{\prime}=\boldsymbol{W}_{\text {odd }}-\boldsymbol{W}_{\text {even }}$.

Obviously, (16) satisfies the CS model, and the sparse recovery algorithm, such as orthogonal matching pursuit (OMP) can be employed to reconstruct $h^{\text {ave }}$ with fewer pilots and higher accuracy. $\boldsymbol{A}$ is called measurement matrix.

\subsection{Pilot pair optimization algorithm}

In channel estimation based on CS theory, in order to make $\boldsymbol{A}$ satisfy restricted isometry property (RIP) [17], a universal approach is to minimize the mutual coherence of $\boldsymbol{A}$, which is denoted as

$$
\begin{gathered}
u(\boldsymbol{A})=\max _{0 \leqslant c<r \leqslant L-1}\left|\boldsymbol{a}_{c}^{\mathrm{H}} \boldsymbol{a}_{r}\right|= \\
\max _{0 \leqslant c<r \leqslant L-1}\left|\sum_{s=1}^{N_{P}} \mathrm{e}^{-\mathrm{j} 2 \pi p_{s}(r-c)} / N\right|
\end{gathered}
$$

where $\boldsymbol{a}_{r}$ and $\boldsymbol{a}_{c}$ are the $r$ th and $c$ th column vector of $\boldsymbol{A}$.

Because $\boldsymbol{A}$ only depends on the pilot location set $\boldsymbol{P}$, so the pilots optimization is to find a pilot location set $\boldsymbol{P}_{\mathrm{opt}}=\arg \min _{\boldsymbol{P}} \mu(\boldsymbol{A})$.

Here, based on the special pilot pair in Section 3.2, a pilot pair optimization algorithm based on dual layer loops iteration is proposed, and is illustrated in Algorithm 1.

Algorithm 1 Pilot pair optimization based on dual layer loops iteration

Initialization:

(i) Variables: number of groups $G_{P}$, and $N_{P}=2 G_{P}$; length of each group $L_{g}$, and $L_{g}=N / G_{P}$; the sub-carries 
set of all group $\boldsymbol{\Gamma}$, the size is $G_{P} L_{g}$, and $\boldsymbol{\Gamma}[m, n]$ is the index of the $n$th carrier of the $m$ th group, the elements of the $m$ th row of $\boldsymbol{\Gamma}$ are $(m-1) L g+1: m L g$; pattern best, pattern_last and pattern_tempt are three vectors that save the current result, the result of the last loop and the tempt result of optimization.

(ii) Pilot pairs: for each group, the first and second carriers are used as pilots. The initial pilot pairs are $(1,2)$, $\left(L_{g}+1, L_{g}+2\right), \ldots,\left((m-1) L_{g}+1,(m-1) L_{g}+\right.$ $2), \ldots,\left(\left(G_{p}-1\right) L_{g}+1,\left(G_{p}-1\right) L_{g}+2\right)$, saved in the pattern_best.

Loop interation:

while (1)

pattern_last $=$ pattern_best;

for $i_{1}=1: G p$

for $i_{2}=\boldsymbol{\Gamma}[i 1,1]: \boldsymbol{\Gamma}\left[i 1, L_{g}\right]$

submit the $\left(\left(i_{1}-1\right) L g+1\right)$ th, and the $\left(\left(i_{1}-1\right) L g+2\right)$ th element of pattern_last with $i_{2}$ and $i_{2}+1$, then get a new pattern pattern_tempt.

According (17), compare the coherence of the measurement matrix of pattern_tempt and pattern_last.

If $\mu($ pattern」tempt $)<\mu($ pattern」last $)$, pattern」last $=$ pattern_tempt.

endif

endfor

endfor

if $\mu($ pattern」best $)==\mu($ pattern」last $)$, break;

else pattern $\_$best $=$pattern $\_$last;

endif

end while

\section{Simulation results}

\subsection{Simulation setting}

Two classical channel models are used: COST 207 RA (rural area) and COST 207 BU (bad urban). The parameters of channel and OFDM system are shown as Table 2 and Table 3, respectively.

Table 2 COST 207 RA/BU channel model

\begin{tabular}{|c|c|c|c|}
\hline Path & Path delay/us & Path gain/dB & Doppler spectrum \\
\hline 1 & $0 / 0$ & $0 /-3$ & Rice/Jakes \\
\hline 2 & $0.4 / 0.4$ & $-2 / 0$ & Jakes/Jakes \\
\hline 3 & $0.4 / 1.0$ & $-10 /-3$ & Jakes/Gauss 1 \\
\hline 4 & $0.6 / 1.6$ & $-20 /-5$ & Jakes/Gauss1 \\
\hline 5 & Null/5.0 & Null/-2 & Null/Gauss2 \\
\hline 6 & Null/6.6 & Null/-4 & Null/Gauss2 \\
\hline
\end{tabular}

Table 3 OFDM system parameter setting

\begin{tabular}{ccccccc}
\hline $\begin{array}{c}\text { System } \\
\text { bandwidth/MHz }\end{array}$ & $\begin{array}{c}\text { Sample } \\
\text { period/ns }\end{array}$ & $\begin{array}{c}\text { Carriers } \\
\text { space/kHz }\end{array}$ & $\begin{array}{c}\text { Carriers } \\
\text { number }\end{array}$ & $\begin{array}{c}\text { Guard } \\
\text { interval }\end{array}$ & $\begin{array}{c}\text { Chanel } \\
\text { code }\end{array}$ & $\begin{array}{c}\text { System } \\
\text { frequency/GHz }\end{array}$ \\
\hline 25.6 & 31.25 & 25 & 1024 & 256 & No & $1-4$ \\
\hline
\end{tabular}

According to the application requirements and channel parameters, the maximum moving speed of the mobile station is $50 \mathrm{~m} / \mathrm{s}$, the normalized Doppler frequency shift $v_{\max }$ is between 0.012 and 0.036 , which satisfies the LTV channel model. Two extreme conditions are selected in simulation, that is, $v_{\max }=0.012$ and $v_{\max }=$ 0.048 , which stand for the "small" and "large" normalized Doppler frequency shift, for RA and BU environment, four kind channels are denoted as RA-small, RA-large, BUsmall, BU-large.

\subsection{Performance comparasion of different methods}

We compare the performance of three different LTV channel estimation methods, including the traditional method in [3], the improved method in [6] and [7], and the new method in this paper. The traditional method and the improved method all require the number of pilots not smaller than the channel length, for the worst case, the number of pilots used in these two methods is $256\left(N_{P}=256\right)$, and both using comb-type pilot pattern with equal space, denoted as "Traditional-256" and "Improved-256". For the new method presented in this paper, we use the pilot design optimization algorithm in Section 4.2 to obtain two optimal pilot patterns with different lengths, $N_{P}=192$ and $N_{P}=256$, denoted as "New-192" and "New-256". The mean square error (MSE) and bit error ratio (BER) of three methods are shown as from Fig. 3 to Fig. 6.

Through the comparison of MSEs and BERs in different channel environment, we can draw the following conclusions: First, with the increase of the number of multipath and Doppler shift, the performance of three methods reduces with different degrees.

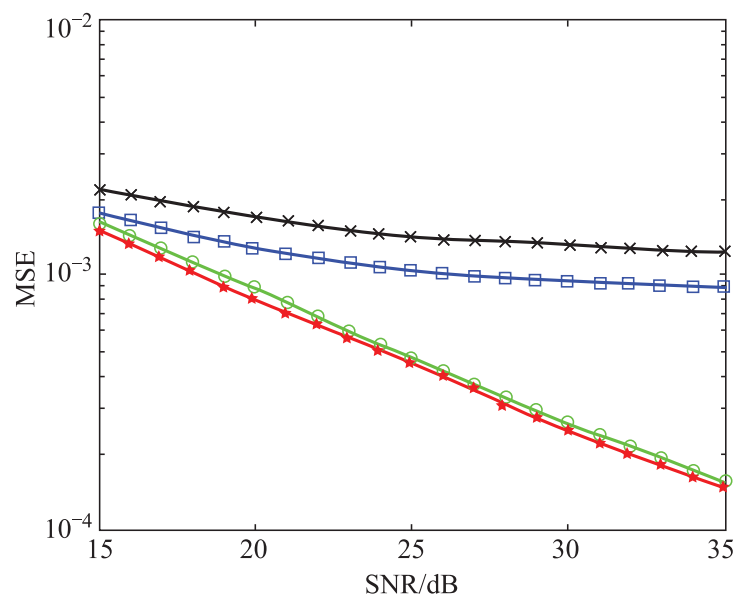

(a) MSE 


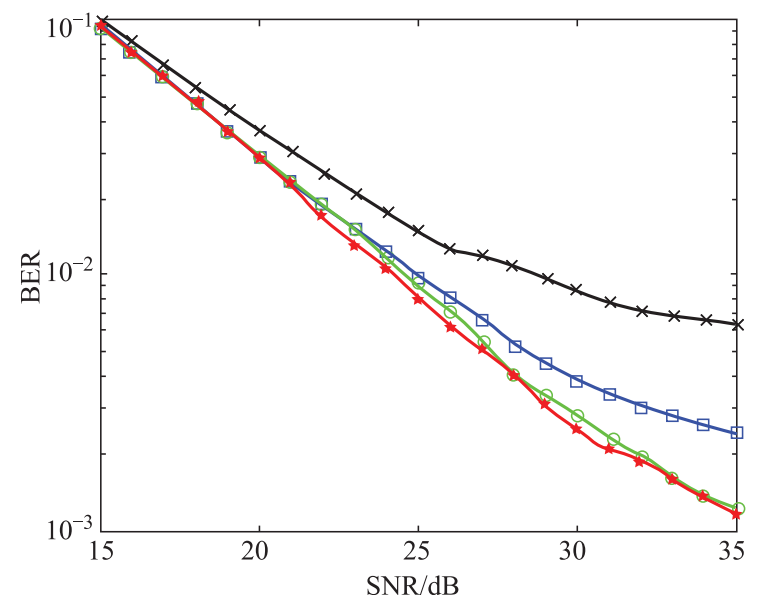

(b) BER

$\rightarrow$ : Tranditional-256; $\square:$ Improved-256;

$\neg$ : New-192; $\rightarrow:$ New-256.

Fig. 3 MSEs and BERs of three methods in COST 207RA-small $\left(v_{\max }=0.012\right)$

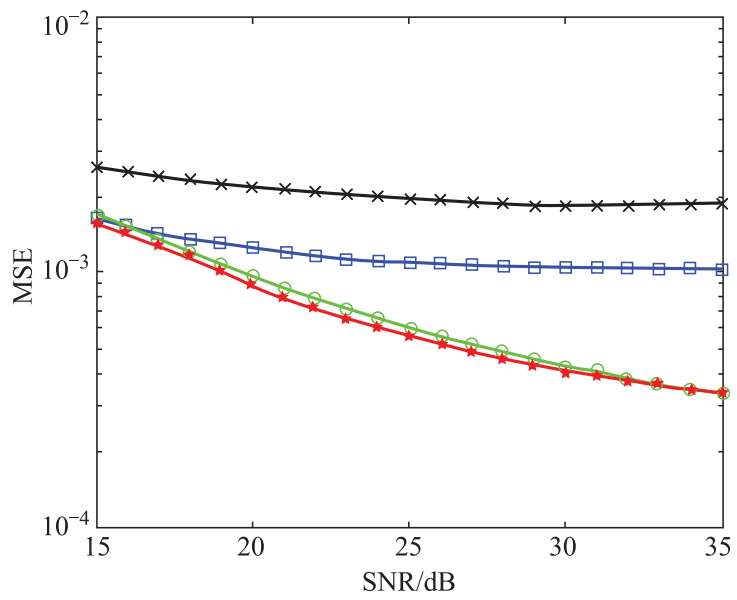

(a) MSE

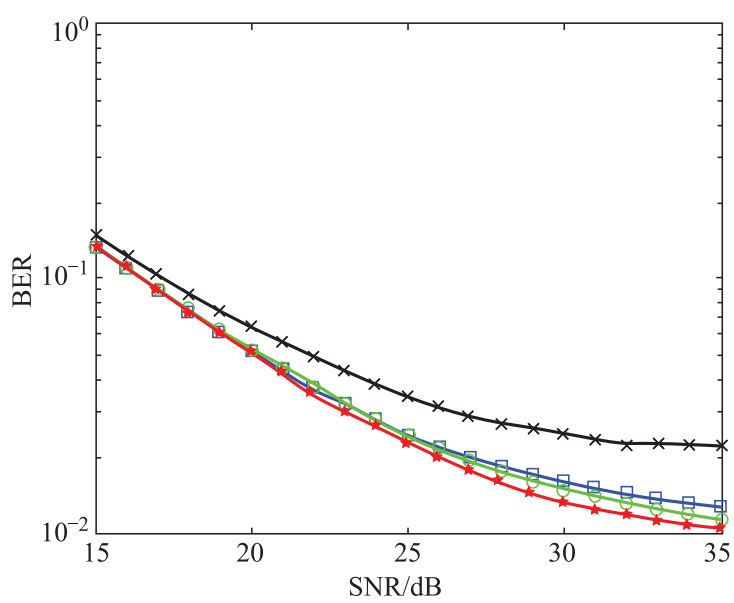

(b) BER

$\star *:$ Tranditional-256; $\square$ : Improved-256;
$\multimap$ : New-192; $\rightarrow:$ New-256.

Fig. 4 MSEs and BERs of three methods in COST 207RA $\left(v_{\max }=\right.$ 0.048 )

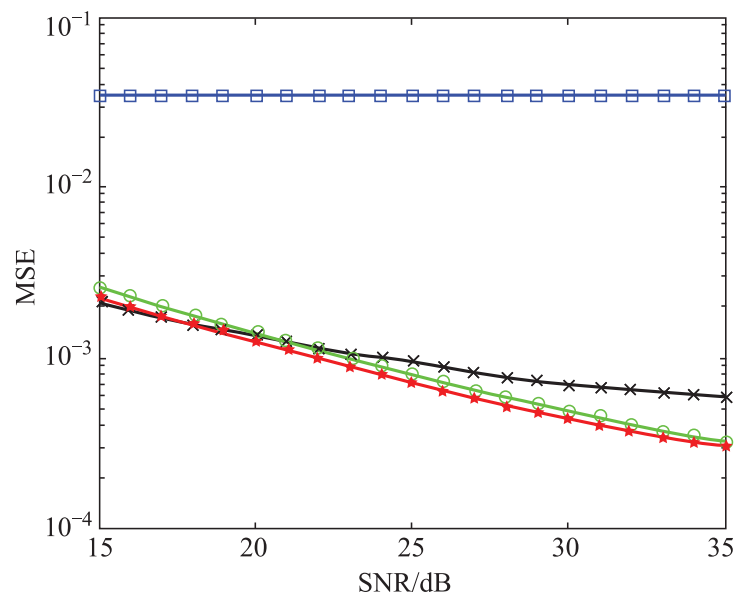

(a) MSE

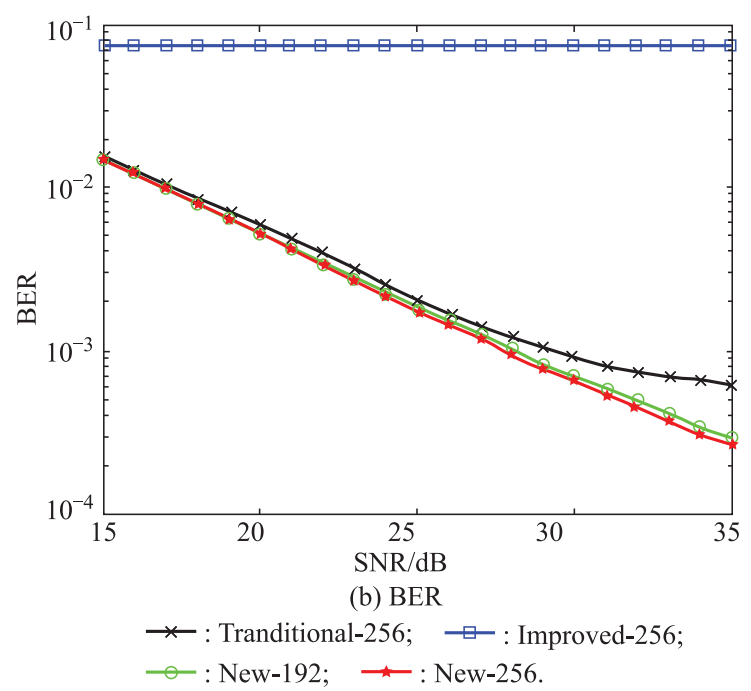

Fig. 5 MSEs and BERs of three methods in COST 207BU ( $v_{\max }=$ 0.012 )

Compared to the other two methods, the change of the traditional LTV method in [3] is not obvious, and it is much more robust to channel, however, whose performance is not the best in these scenarios. Second, for the improved method in [6] and [7], the effect of ICI is both taken into consideration during pilot design and equalization, so in the COST 207 RA channel, its performance is much better than the traditional method. However, the result in COST $207 \mathrm{BU}$ channel is very poor, so it is only suitable for simple multipath channel with low delay. Third, the performance of the new method is much better than the traditional method, for all channel environments except the one with large delay and Doppler shift. Furthermore, compared with the latter, the former uses fewer pilots, and saves about 1/4. Compared with the improved method in [6] and [7], the result of the new method is improved with different degrees, especially for COST 207 BU channel. Finally, due to using the sparse reco- 
very algorithm and pilot optimization algorithm, the new method does not have strict requirement on the number of pilots, and the performance difference between $N_{P}=192$ and $N_{P}=256$ is not significant, so it also improves the utilizing rate of frequency. Obviously, not only for the performance, but also the frequency utilization, the new method is all the best among the current channel estimation methods based on LTV.

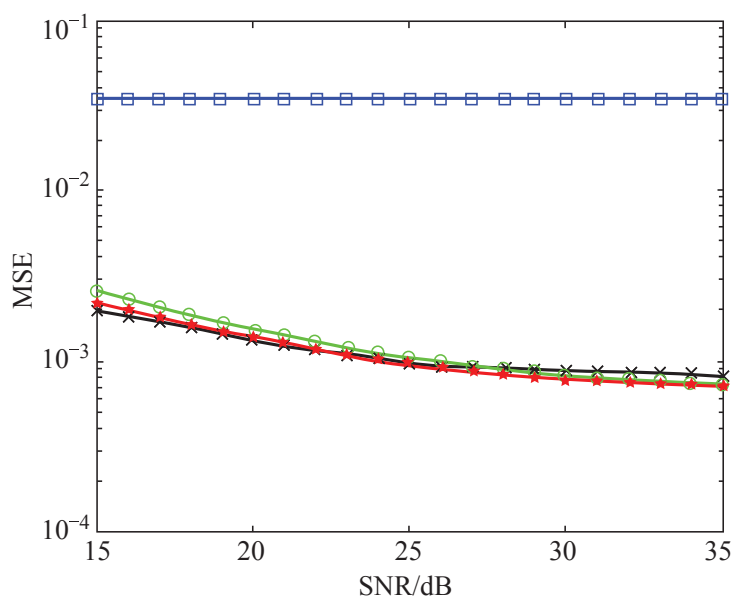

(a) MSE

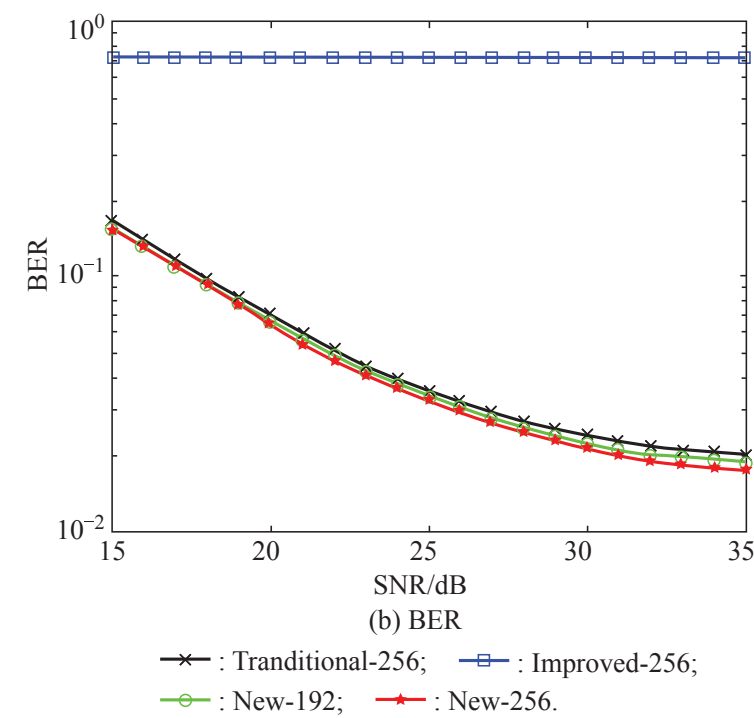

Fig. 6 MSEs and BERs of three methods in COST 207BU ( $v_{\max }=$ 0.048)

\section{Conclusions}

Base on the principle of the LTV model and the ICI, we design a new pilot pattern with ICI self-elimination. Then, a new OFDM channel recovery model based on CS is reconstructed. Moreover, we also design a pilot pair optimization algorithm to realize the minimization of mutual coherence of the measurement matrix. In this paper, the experiments are limited to four kinds of simulated communication environments, and results show the good performance of the new methods among all current methods based on the LTV model. We will investigate its effect on more realistic OFDM systems in the future.

\section{References}

[1] WU J X, FAN P Z. A survey on high mobility wireless communications: challenges, opportunities and solutions. IEEE Access, 2016, 4(1): 450-479.

[2] LIU Y S, TAN Z H, HU H J. Channel estimation for OFDM. IEEE Communications Surveys \& Tutorials, 2014, 16(4): $1891-1908$.

[3] MOSTOFI Y, COX D C. ICI mitigation for pilot-aided OFDM mobile systems. IEEE Trans. on Wireless Communications, 2005, 4(2): 765-774.

[4] KWAK K, LEE S, MIN H. New OFDM channel estimation with dual-ICI cancellation in highly mobile channel. IEEE Trans. on Wireless Communications, 2010, 9(10): $3155-$ 3165.

[5] XIE Y S, WANG M L, ZHOU L L. Windowed channel estimation of OFDM systems in linear time-varying channels. Journal of South China University of Technology (Natural Science Edition), 2013, 41(5): $43-47$.

[6] TAO C, QIU J H, LIU L. A novel OFDM channel estimation algorithm with ICI mitigation over fast fading channels. Radio Engineering, 2010, 19(2): 347-355.

[7] DING Y, OUYANG S, XIE Y L, et al. Time-varying channel estimation in OFDM systems based on ICI self-cancellation of pilots. Systems Engineering and Electronics, 2014, 36(8): 1632 - 1637. (in Chinese)

[8] VUOKKO L, KOLMONEN V M, SALO J. Measurement of large-scale cluster power characteristics for geometric channel models. IEEE Trans. on Antennas Propagation, 2007, 55(11): $3361-3365$.

[9] GAO Z, ZHANG C, WANG Z C. Priori-information aided iterative hard threshold: a low-complexity high-accuracy compressive sensing based channel estimation for TDS-OFDM. IEEE Trans. on Wireless Communications, 2015, 15(1): $242-$ 252.

[10] BAIJWA W U, SAYEED A M, NOWARK R. Learning sparse doubly-selective channels. Proc. of Conference on Communication, Control \& Computing, 2008: 575-582.

[11] CHENG P, CHEN Z, RUI Y. Channel estimation for OFDM systems over doubly selective channels: a distributed compressive sensing based approach. IEEE Trans. on Communications, 2013, 61(10): $4173-4185$.

[12] QIN Q B, LIU G, GONG B. Structured distributed compressive channel estimation over doubly selective channels. IEEE Trans. on Broadcasting, 2016, 99(4): $1-11$.

[13] BARBU O E, MANCHON C N, ROM C. OFDM receiver for fast time-varying channels using block-sparse Bayesian learning. IEEE Trans. on Vehicular Technology, 2016, 99(4): 1-5.

[14] PAKROOH P, AMINI A, MARVASTI F. OFDM pilot allocation for sparse channel estimation. EURASIP Journal on Advances in Signal Processing, 2012, 2012(1): 59.

[15] QI C H, YUE G S, WU L N. Pilot design schemes for sparse channel estimation in OFDM systems. IEEE Trans. on Vehicular Technology, 2015, 64(4): 1493 - 1506.

[16] WANG H, GUO Q, ZHANG G X. Pilot pattern optimization for sparse channel estimation in OFDM systems. IEEE Communication Letters, 2015, 19(7): 1233-1236.

[17] CANDES E J, TAO T. Decoding by linear programming. IEEE 
Trans. on Information Theory, 2005, 51(12): 4203-4215.

[18] DU Z, SONG X G, CHENG J L. Maximum likelihood based channel estimation for macro cellular OFDM uplinks in dispersive time-varying channels. IEEE Trans. on Wireless Communication, 2011, 10(1): 176-187.

\section{Biographies}

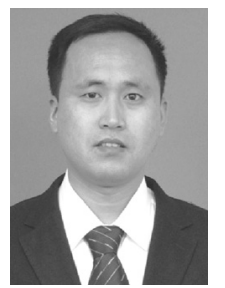

HU Jiansheng received his Bachelor and Master degrees in Telecommunications College, Air Forces Engineering University in 2006 and 2009 respectively. He is a Ph.D. candidate at School of Electronics and Information, Northwestern Polytechnical University. His research interests are unmanned aerial vehicle (UAV) data link, channel estimation and the signal process based on compressive sensing.

E-mail: hujiansheng121@163.com

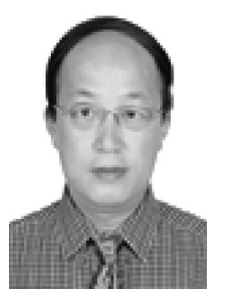

SONG Zuxun received his Bachelor, Master, and $\mathrm{Ph} . \mathrm{D}$. degrees from School of Electronics and Information, Northwestern Polytechnical University in 1986, 1989, and 2004 respectively. He now is a professor at the School of Electronics and Information, Northwestern Polytechnical University. His research interests include signal information acquisition and processing, overall design of microwave communication link, and electro magnetic compatibility design and diagnosis. E-mail: zxsong@nwpu.edu.cn

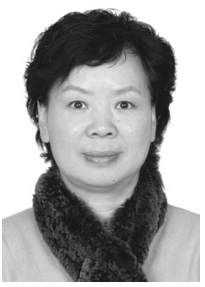

GUO Shuxia received her Bachelor degree from Shenyang Ligong University in 1986, Master and $\mathrm{Ph} . D$. degrees from Northwestern Polytechnical University in 2002 and 2008 respectively. She now is an associate professor at the School of Electronics and Information, Northwestern Polytechnical University. Her research interests include signal information acquisition and processing and electromagnetic environment simulation.

E-mail: guoshuxia0223@163.com

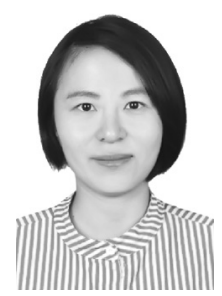

ZHANG Qian received her Bachelor and Master degrees from Engineering University of Chinese People's Armed Police Force in 2007 and 2010 respectively. She is currently working as a teaching assistant at Engineering University of Chinese People's Armed Police Force. Her research interests are wireless sensor network and network security.

E-mail: zhangqianjy@163.com

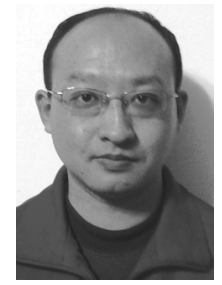

SHUI Dongdong received his Bachelor and Master degrees from Engineering University of Chinese People's Armed Police Force in 2001 and 2004 respectively. He is currently working as teaching assistant at Engineering University of Chinese People's Armed Police Force. His research interests are UAV mission planning and military communication. E-mail: 1636652148@qq.com 